\title{
ANALYSIS OF THE SOLAR GRANULATION IN THE OPACITY MINIMUM REGION
}

\author{
Serge Koutchmy \\ Institut d'Astrophysique CNRS, 98bis Bd Arago, F-75014 Paris, France \\ and \\ NSO-Sacramento Peak, Sunspot, 88349 NM, USA
}

\begin{abstract}
An observing program in the spectral region of the opacity minimum region near $1650 \mathrm{~nm}$ was started at NSO/SP-VTT many years ago and few results were presented. Thanks to an image processing unit connected to a rather conventional TV camera, IR images taken at $1650 \mathrm{~nm}$ can be displayed on the TV monitor and images stored on a VCR for subsequent comparison with corresponding images in the visible spectrum. This rather qualitative analysis is substantiated thanks to results coming from one-dimensional time series obtained with an IR pin-hole spectrophotometer. Power spectra of the o.m.r. granulation as well as its temporal behaviour are compared with results obtained using the same method in the optical region of the solar spectrum. Noticeable differences are emerging, including the reduced effect of 5-min oscillations.
\end{abstract}

\section{Introduction}

The solar atmosphere radiative opacity reaches its absolute minimum in the $1650 \mathrm{~nm}$ spectral region of the continuum, see Vernazza et al. 1981, which is also a region of excellent Earth atmosphere spectral transmission, making groundbased observations at this particular wavelengths of high quality. Unfortunately, the specificity of infrared technology and method, see Turon and Léna, 1973, has limited the progress which has been anticipated. Indeed the access of this solar opacity minimum region (o.m.r.) offers a great potential for the analysis of the structures of the solar atmosphere, starting with granulation. Here, we will concentrate on the "quiet" granulation (outside of active region) analysed during years of the solar minimum (1975-76 and 1987-88), see also Turon, 1975.

We first notice that the contribution function deduced for the homogeneous standard solar atmosphere of Vernazza et al., 1981, shows that in the o.m.r. at $1650 \mathrm{~nm}$ the radiation comes from layers deeper by approximately $40 \mathrm{~km}$ than the radiation of the optical continuum in the 500-600 $\mathrm{nm}$ region. However, although this value is rather small compared to the typical "scale" of granules, see Bray et al., 1984, it is interesting to notice also the extension of the contribution function : in the o.m.r. the radiation is coming from a rather limited vertical extension layer, contrary to the optical case where a substantial part of the radiation is still produced in the upper part of the "continuum" photosphere where 5-min oscillations start to become of considerable amplitude, see Keil, 1980. Taking into account the results of Keil extrapolated to the deepest layers, 5-min oscillations should become there evanescently small and, accordingly, convective motions should be dominant (especially in vertical direction). Concerning the analysis of the optical granulation, 5-min oscillations have been shown to give a substantial contribution, see Koutchmy and Lebecq, 1986, and Title et al., 1989, which corresponds 
to temperature fluctuations not to be confounded with the convective of origin temperature fluctuations of the granulation. Finally, let us notice that intensity modulations (the "contrast") corresponding to these temperature fluctuations are considerably smaller in o.m.r. which corresponds to the infrared (IR) part of the solar spectrum; a $100 \mathrm{~K}$ temperature fluctuation correspond to a $8.5 \%$ intensity variation at $500 \mathrm{~nm}$ and only $3.5 \%$ at $1690 \mathrm{~nm}$.

There exists no obvious imager in the IR The linear array built by T \& L, 73 to perform there o.m.r. observations at the Mac Math telescope failed to produce useful 2D-images; their best results have been deduced using a single detector and telescope scanning. Furthermore, a telescope of large enough aperture should be used in order to limit the influence of the diffraction, the wavelength of the o.m.r. being of order of 3 times longer than the optical one. Fortunately, an other limitation due to the Earth Atmosphere smearing and image motion is improved in the IR.

2. Imaging of granules in the o.m.r. and brightness distribution

Following the experience of T \& L, 73, I started to image the IR granulation at the VTT of NSO/S.P. in 1975, during the sunspot minimum. Typical matrices of $32 \times 32$ were recorded in $43 \mathrm{sec}$ of time by scanning the center of the Sun using a computer controlled motion of one of the primary plan mirror of the heliostat and a specially designed pin-hole photometer (unsuccessful tests were performed in 1972 at the Pic du Midi Observatory and in 1974, at the $1 \mathrm{~m}$ aperture Crimean Astrophysical Observatory) working in the o.m.r. with different passbands (from $100 \mathrm{~nm}$ to $3 \mathrm{~nm}$ ), see Koutchmy et al., 1977. Time series of several tens of images were obtained showing convincingly the improvement of the seeing in IR. However, images are now obtained more easily with an IR vidicon equiped with a video-digitizer. The first movies showing the IR granulation have been obtained during the summer of 1988 , with a conventional IR tube and TV camera. Time sequences of IR nearly diffraction-limited granulation images were obtained at a slow rate of 2 images of $512 \times 512 \mathrm{px}^{2}$ per sec of time, see Figure 1, through a $110 \mathrm{~nm}$ interference filter centered at $1650 \mathrm{~nm}$; the signal level is adjusted, as well as the signal-noise ratio, with both the target voltage and the video amplifiers; unfortunately, the sensitivity of the tube is inhomogeneous by up to $40 \%$ over the field and the precision of digitization limited by the 8 bits $A / D$ converter.

Before a time series is started, a gain table is built using a flat field and stored in

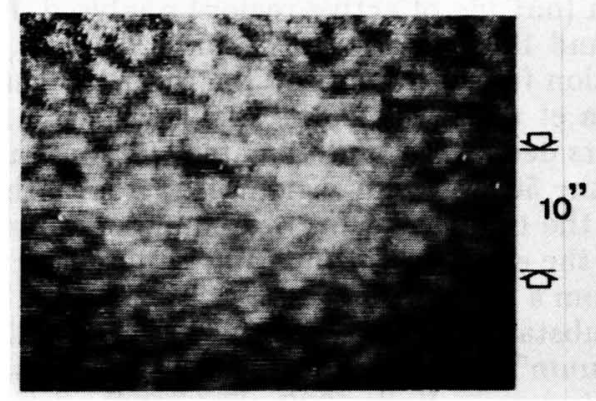

t2 $2 \mathrm{mn} 02 \mathrm{sec}$

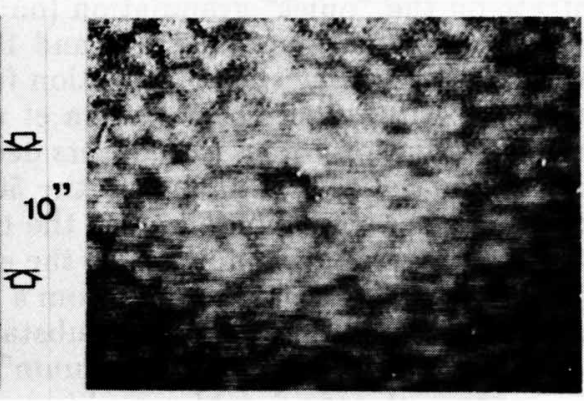

t1 $1 \mathrm{mn} 31 \mathrm{sec}$

Figure 1. Direct images of the $1650 \mathrm{~nm}$ o.m.r. granulation taken from a slow rate video-movie. Each image is processed in real time to remove the px to px variation of sensitivity. 
the SUN computer which operates a VICOM multi-processor; accordingly, a pixel to pixel scaling is performed after each digitization, i.e. 2 times per sec, thanks to a loop giving each time the result on a central monitor; processed images are stored on a VCR. More sophisticated algorithm has been also used to look at umbral dots, etc. In this experiment, a second channel was simultaneously recorded in real time, to show the optical granulation with a conventional video-CCD camera fed by a splitter. Although both movies are obviously showing the same bright and dark features, the behaviour of the images is quite different, partly because the effect of the Earth atmospheric induced seeing effects are different for each channel and partly because a real difference exists in brightness distribution.

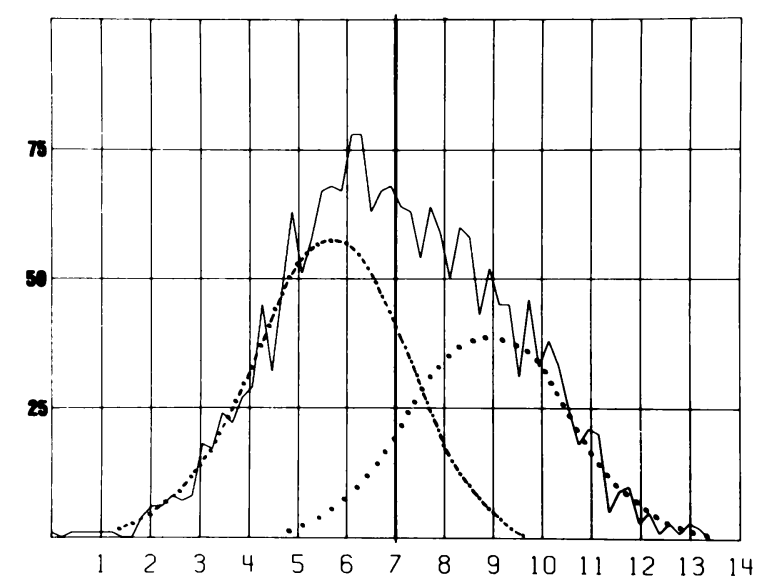

Figure 2. Histogram analysis of the brightness distribution of the the o.m.r. granulation. One unit on the abscissa corresponds to a $1 \%$ intensity variation. The bin width is $10^{-3}$ of the average intensity (with abscissa value 7).
In the visible region, it is well known that even with a good image quality, when the uncorrected RMS reaches 5 to $7 \%$, the histogram of brightness fluctuations is nearly gaussian, see Pravdjuk et al. 1974. We performed the same analysis with data taken in the o.m.r. at $1750 \mathrm{~nm}$ (see further) and, surprisingly, the histogram shows clearly, see Figure 2, a skewed bidistribution which can be perceived in the optical region only when a very high quality picture (RMS $\geq$ $9 \%$ ) is used.

\section{Results of the statistical analysis of granules in o.m.r.}

Using computer controlled scans of the same region at different $\mu=\cos \theta$ values, a great amount of data representing the brightness distribution of the quiet Sun o.m.r. granulation has been collected and analysed; a similar study has also been performed at $600 \mathrm{~nm}$, see Koutchmy and Lebecq, 1986. The typical digitization rate was $100 \mathrm{px}-\mathrm{sec}^{-1}$, with a 14 bits.px ${ }^{-1}$ precision; linear synchronous detection of the modulated at $1100 \mathrm{~Hz}$ signal was achieved, resulting in a $\mathrm{S} / \mathrm{N}$ ratio of 2000 in the o.m.r. (at the center of the Sun with a broad filter) and of 175 at $600 \mathrm{~nm}$. A round pin-hole of .75 arcsec effective diameter and a .19 arcsec sampling interval was systematically used; the corresponding smearing function and MTF have been evaluated both theoretically and with numerous scans of the extreme-limb, see Koutchmy et al. 1977 and Koutchmy, 1977. We first computed the RMS (after subtraction of the best $2^{d}$ order polynomial from each scan of at least 200 arcsec length), the auto-correlation function, power-spectra, etc.. Scans were also cross-correlated, to evaluate the "life-time" of granules and even, using the drift of the center of gravity of the cross-correlation function, the solar rotation. Data collected at the VTT of NSO/S.P. are significantly better than the best results coming from the Mac Math telescope : higher RMS, good reproducibility of the scans, significant temporal cross-correlation up to $16 \mathrm{~min}$, etc.. The typical values of the corrected for the foreshortening $\left(\sim \mu^{-1 / 2}\right)$ RMS of ob- 
served intensity fluctuations in the o.m.r. at $1750 \mathrm{~nm}$ are : \pm 2.04 at $\mu=1$; \pm 2.02 at $\mu=.8$ and \pm 1.96 at $\mu=.7$. Only a slight center-limb effect is perceived on this range of values of $\mu$. The 2-D power spectrum has also been computed and even corrected for the smearing; compared to the spectrum obtained at $600 \mathrm{~nm}$, more power appears in the high spatial frequencies region (although results are very sensitive to the values of the correction) and, more significantly, far less power appears in the low frequencies region, see Figure 3.

Finally, the cross-correlation analysis permits the determination of the "lifetime" of granules. Exactly the same procedure was used at $600 \mathrm{~nm}$ and in the o.m.r. giving there a definitly smaller value $(3.25 \mathrm{~min}$ instead of $6 \mathrm{~min})$ of the decrement of the best exponential function which fits the data in each case. This strongly suggests a smaller characteristic time of evolution of the o.m.r. granulation, consistent with an increase of the convective velocities with the depth, see Keil, 1980. However, it is not clear how the 5-min oscillations clearly affecting the intensity fluctuations at $600 \mathrm{~nm}$, see Koutchmy and Lebecq, 1986, bias the results. Title et al. 1989 suggested a method of correction which seems to work for comparing the "quiet" and the "active" region granulation. I performed a spectral analysis of the o.m.r. time series using different methods of averaging : the 5-min oscillations are clearly of smaller amplitude in the o.m.r. and even at large spatial scales they barely emerge from the noise, see Figure 4.

\section{References}

Bray, R.J., Loughhead, R.E. and Durrant, C.J. : 1984, “The Solar Granulation”, Cambridge Univ. Press.

Keil, S.L. : 1980, Astron. Astrophys. 82, 144

Koutchmy, S., Koutchmy, O. and Kotov, V. : 1977, Astron. Astrophys. 59, 189

Koutchmy, S. and Lebecq, C. : 1986, Astron. Astrophys. 169, 323

Title, A.M., Tarbell, T.D., Topka, K.P., Ferguson, S.H. and Shine, R.A. and the SOUP team : 1989, Ap.J. in press

Turon, P. : 1975, Solar Phys. 41, 271

Turon, P.J. and Léna, P. : 1973, Solar Phys. 30,3

Vernazza, J.E., Avrett, E.H. and Loeser, R. : 1981, Ap.J. Suppl. 4도 635

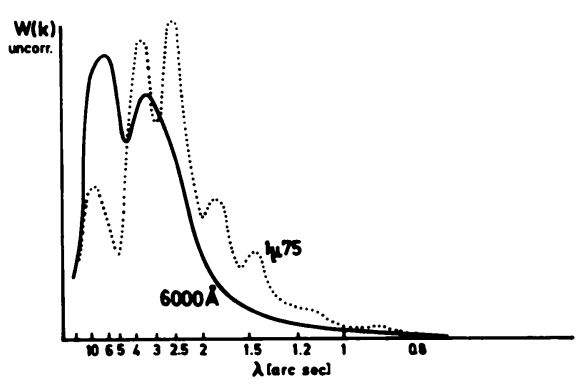

Figure 3. 2-D power spectra per unit surface, computed for the o.m.r. granulation (dotted line) and the $600 \mathrm{~nm}$ granulation (full line), uncorrected and unsmoothed. The small-scale features are due to statistical fluctuations, but the general behaviour is significant.

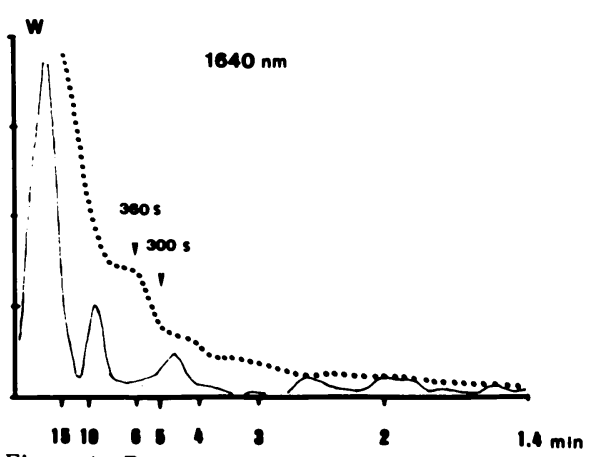

Figure 4. Power spectra of the temporal brightness variation of the o.m.r. granulation. The dotted line is the average of 1100 spectra computed for different points on the Sun from a 46 min time sequence, while the full line has been computed using the brightness values averaged over a 206 arcsec length. The repetition time of each scan is $42.8 \mathrm{~s}$. 